\title{
Efficacy, Safety and Cost Effectiveness of Amitriptyline and Pregabalin in Patients with Diabetic Peripheral Neuropathy
}

\author{
V. SANKAR* ${ }^{*}$ ANU ELIZABETH OOMMEN ${ }^{1}$, ANU THOMAS $^{1}$, J. V. NAIR ${ }^{1}$ AND J. S. JAMES ${ }^{1}$ \\ Department of Pharmaceutics, ${ }^{1}$ Department of Pharmacy Practice, PSG College of Pharmacy, Coimbatore-641 004, India
}

Sankar, et al: Comparison of Amitriptyline and Pregabalin in Diabetic Neuropathy Patients

\begin{abstract}
The tricyclic antidepressant, amitriptyline and the anticonvulsant, pregabalin are the most frequently used medications in general practice for diabetic peripheral neuropathy. This study compares the effectiveness of amitriptyline vs. pregabalin using neuropathic pain symptom inventory and visual analogue scale, the incidence of adverse drug reactions and the cost effectiveness. Effectiveness was measured using visual analogue scale and neuropathic pain symptom inventory scores both before and after therapy. Adverse drug reactions were documented after the therapy and cost effectiveness was measured using incremental cost effectiveness analysis ratio. Both drugs showed obvious pain relief. Visual analogue scale mean difference scores showed statistically significant difference in efficacy for amitriptyline and pregabalin. Incidences of adverse drug reactions were more or less similar in terms of magnitude. Furthermore the cost of amitriptyline is less than that of pregabalin. Hence we concluded that amitriptyline was a better choice of therapy than pregablin because it was well tolerated and has a lower cost for the additional effectiveness achieved.
\end{abstract}

Key words: Diabetic neuropathy, diabetes mellitus, amitriptyline, pregabalin, visual analogue scale, neuropathic pain symptom inventory

Diabetes mellitus is defined as a chronic condition caused by an absolute lack of insulin or relative lack of insulin as a result of impaired insulin secretion and action. Globally, the prevalence of diabetes among all the age groups was estimated to $2.8 \%$ in 2000 and projected to increase to $4.4 \%$ by 2030 . The incidence of diabetes mellitus is now an epidemic, with alarming increase in prevalence in both adults and children. Its hallmark clinical characteristics are symptomatic glucose intolerance resulting in hyperglycaemia and alterations in lipid and protein metabolism ${ }^{[1]}$. Chronic hyperglycaemia can lead to damage and potential failure of various organs, including the eyes, heart, kidney, blood vessels and nerves ${ }^{[2]}$.

Diabetic peripheralneuropathy(DPN) is amicrovascular complication of diabetes mellitus represented by diffuse, symmetrical, and length dependent injury to the peripheral nerves. DPN occurs in distal portions of lower extremities. Symptoms include hypersensitivity to light touch and severe burning sensation ${ }^{[3]}$. It affects $16.2 \%$ of patients with diabetes of which $12.5 \%$ is unreported and $39 \%$ is untreated. It has a major implication on quality of life, morbidity, and cost. Treatment includes antidepressants, anticonvulsants, opioids, capsaicin, membrane stabilizers, and analgesics ${ }^{[4]}$.

The tricyclic antidepressant, amitriptyline and the anticonvulsant, pregabalin are the most frequently used medications in general treatment of DPN. Both medications differ in cost, the latter being more expensive. A randomized double blind clinical trial conducted by Bhansal et al. ${ }^{[4]}$ has compared amitriptyline $(\mathrm{n}=22)$ and pregabalin $(\mathrm{n}=22)$ at average doses of 16 and $218 \mathrm{mg} / \mathrm{d}$, respectively. This study found that there is no significant difference in pain relief between both groups but concluded that pregabalin is a good alternative for the treatment of DPN, especially in those who cannot tolerate amitriptyline ${ }^{[4]}$. In the network meta-analysis, which compared efficacy

This is an open access article distributed under the terms of the Creative Commons Attribution-NonCommercial-ShareAlike 3.0 License, which allows others to remix, tweak, and build upon the work non-commercially, as long as the author is credited and the new creations are licensed under the identical terms

Accepted 08 June 2017 Revised 18 February 2017

Received 30 October 2016

Indian J Pharm Sci 2017;79(4):646-650 
and safety of six antidepressants and anticonvulsants in DPN conducted by Neelima et al. demonstrates that pregabalin precedes amitripyline based on most favourable balance between risk and benefit ${ }^{[5]}$. Thus, there is a need to highlight the superior drug, which provides meaningful pain relief and is generally well tolerated in terms of occurrence of adverse drug reactions (ADRs). Hence the present study was aimed to compare the effectiveness of amitriptyline and pregabalin using neuropathic pain symptom inventory (NPSI) and visual analogue scale (VAS) and to find the incidence of ADRs and the cost effectiveness.

A six month (Jan-June 2015) comparative prospective observational study was conducted at a tertiary care hospital. Patients of either gender aged between 18 and $75 \mathrm{y}$ and attending the outpatient or inpatient departments of endocrinology, general medicine, and physical medicine and rehabilitation of a tertiary healthcare hospital with DPN were enrolled for the study. Patients with type 1 or type 2 diabetes mellitus who were treated with either oral hypoglycemic agents (OHAs) or insulin or both were selected. Those who were newly or previously treated with amitriptyline $(10 \mathrm{mg})$ or pregabalin $(75 \mathrm{mg})$ were recruited for the study. Patients were excluded if they had any evidence of cognitive impairment, recent cardiac or cerebral ischemic event, or other causes of neuropathy. Patients with a history of renal disease, liver failure, epilepsy, psychiatric illness, malignancy, dependence or abuse of alcohol or other recreational drugs were excluded from the study. Seventy patients were recruited, of which 35 were on amitriptyline $10 \mathrm{mg}$ once daily and the other 35 subjects were on pregabalin $75 \mathrm{mg}$ once daily for $4 \mathrm{w}$ or more. NPSI and VAS scores were documented before and after therapy. Additional demographic and clinical details were recorded on a data collection form (details collected were chief complaints, past medical history, past medication history, general examination parameters, social habits, family history, laboratory tests, diagnosis, and drug treatment chart). ADRs were recorded during follow up appointments at the hospital or by phone call.

The primary objective of the study was to compare the effectiveness of amitriptyline and pregabalin considering the decline in pain scores using NPSI and VAS. NPSI allows discrimination and quantification of the five distinctly relevant dimensions of neuropathic pain, which are superficial pain, deep pain, paroxysmal pain, evoked pain, and paraesthesia or dysaesthesia. It comprises 12 questions each of which scores pain on a scale of 0 to 10, 0 being "no pain" and 10 being the "worst pain imaginable". VAS measures the general pain the patient experiences, which ranges from 0 to 10,0 being "no pain" and 10 being "worst pain". NPSI was developed and validated over a period of $3 \mathrm{y}$ in consecutive patients recruited from five pain clinics in France ${ }^{[6]}$.

A checklist for ADR documentation was designed with respect to the ADR components somnolence, day time sleepiness, dizziness, constipation, peripheral edema, flu like symptoms for pregabalin and for amitriptyline dizziness, constipation, somnolence, day time sleepiness, difficulty in urination, postural hypotension, dry mouth were noted for recording adverse events during the follow up.

Cost effectiveness analysis is a technique used to aid in decision making between alternatives, when the cost is measured in monetary terms but the consequences are measured in natural unit changes in health. It is analysed using Incremental cost effective analysis ratio (ICER). The price per tablet of amitriptyline ranges from $₹$ 1.5-1.7, whereas pregabalin ranges from ₹ 8.78-9.24.

Paired t-test (graph pad prism version 5) was used to compare VAS and NPSI scores before and after therapy. Unpaired ' $t$ ' test was used to compare VAS and NPSI scores of pregabalin and amitriptyline. Two tailed tests and $\mathrm{P}<0.05$ established statistical significance. ICER was calculated using the following Eqn. ${ }^{[7]}$, difference in cost $(\mathrm{A}-\mathrm{B})$ /difference in benefit $(\mathrm{A}-\mathrm{B})$.

Pain is an unpleasant and highly subjective experience. A person's perception of pain is affected by environmental, emotional, cultural, spiritual and cognitive factors. Unrelieved chronic pain, affects not only physical comfort but also a person's psychological and social well-being. Hence, deciding a better alternative depends on the assessment and quantification of pain. The readiness of the patient and healthcare provider to use a medication depends on the extent of the expected benefit of the remedy balanced by the magnitude of the risk and seriousness of possible unwanted effects.

All demographic and clinical characteristics were given in Table 1. A total of 70 subjects were recruited out of which 35 patients were on amitriptyline $10 \mathrm{mg}$ once a day and the other 35 were on pregabalin $75 \mathrm{mg}$ once a day. Antidiabetic therapies given to the recruited patients are listed in Table 2.

Two tailed tests and $\mathrm{P}<0.05$ was used to confirm 
TABLE 1: DEMOGRAPHICS AND CLINICAL CHARACTERISTICS

\begin{tabular}{lcc}
\hline Parameter & Amitriptyline $(\mathrm{n}=\mathbf{3 5})$ & Pregabalin $(\mathrm{n}=\mathbf{3 5})$ \\
\hline Male & \multicolumn{1}{c}{ Sex } \\
Female & $15(42.85 \%)$ & $22(62.85 \%)$ \\
Age $($ in y) & $20(57.14 \%)$ & $13(37.14 \%)$ \\
& $55.85 \pm 9.96$ & $57.62 \pm 8.977$ \\
\hline Hypertensive on treatment & Comorbidities & \\
Others & $9(25.71 \%)$ & $14(40 \%)$ \\
& $13(37.14 \%)$ & $16(45.71 \%)$ \\
\hline Fasting blood sugar $(\mathrm{mg} / \mathrm{dl})$ & Clinical parameters & $180.82 \pm 92.122$ \\
Random blood sugar $(\mathrm{mg} / \mathrm{dl})$ & $147.09 \pm 55.206$ & $193.42 \pm 62.457$ \\
Postprandial blood sugar $(\mathrm{mg} / \mathrm{dl})$ & $216.5 \pm 143.379$ & $252.05 \pm 111.249$ \\
Glycosylated hemoglobin $(\%)$ & $222.78 \pm 73.786$ & $9.25 \pm 2.628$ \\
\hline
\end{tabular}

TABLE 2: ANTIDIABETIC THERAPY GIVEN TO PATIENTS

\begin{tabular}{lcc}
\hline Parameter & Amitriptyline $\mathbf{( n = 3 5 )}$ & Pregabalin $(\mathbf{n}=\mathbf{3 5})$ \\
\hline Insulin & Diabetes treatment & $6(17.14 \%)$ \\
OHAs & $19(54.28 \%)$ & $19(54.28 \%)$ \\
Both & $16(45.71 \%)$ & $10(28.57 \%)$ \\
OHAs & & \\
Sulfonyl ureas & $8(22.85 \%)$ & $5(14.28 \%)$ \\
Metformin & $22(62.85 \%)$ & $18(51.42 \%)$ \\
Repaglinide & $2(5.71 \%)$ & 0 \\
Acarbose & 0 & 0 \\
& Combination of OHAs & \\
Vidagliptin and metformin & 0 & $1(2.8 \%)$ \\
Glimepiride and metformin & $1(2.85 \%)$ & $1(2.8 \%)$ \\
Gliclazide and metformin & $4(11.42 \%)$ & $4(11.42 \%)$ \\
Acarbose and metformin & $2(5.71 \%)$ & 0 \\
& Insulin & $8(22.85 \%)$ \\
Mixtard & $1(5.71 \%)$ & $2(5.71 \%)$ \\
Actrapid & $1(2.85 \%)$ & $5(14.28 \%)$ \\
Huminsulin & $2(8.57 \%)$ & $2(5.71 \%)$ \\
Insugen N & $0(0 \%)$ & $1(2.85 \%)$ \\
Lupinsulin & $0(0 \%)$ &
\end{tabular}

OHAs: oral hypoglycaemic agents

statistical significance. Effectiveness scores were shown in Table 3. VAS and NPSI scores before and after therapy were compared using paired t-test. There was a statistically significant decrease in VAS and NPSI scores after therapy for both amitriptyline and pregabalin. Mean difference VAS and NPSI scores for amitriptyline and pregabalin were compared using unpaired t-test. It was estimated that there was a significant difference in the VAS scores of amitriptyline and pregabalin. The mean difference VAS score for amitriptyline 6.34 was higher than that of pregabalin 5.7. Both amitriptyline and pregabalin separately showed significant pain relief when comparing the VAS and NPSI pain scores before and after therapy. There was significant difference between VAS mean difference scores of amitriptyline 6.34 and pregabalin
5.7 but there was no significant difference between NPSI mean difference scores of the same.

Among 35 subjects taking amitriptyline, ADRs were reported by 11 individuals (31.42\%) and among 35 subjects taking pregabalin, ADRs were reported by 12 individuals (34.28\%). Table 4 lists the ADRs recorded and their incidence. Day time sleepiness (9) was the most common ADR reported among patients taking 10 mg amitriptyline followed by dizziness (7), dry mouth (3), and somnolence (2). Similarly, among patients taking $75 \mathrm{mg}$ pregabalin day time sleepiness was the most common ADR followed by dizziness (5) and peripheral oedema (2). In terms of magnitude and level of gravity of the incidence of ADRs for amitriptyline and pregabalin are relatively similar and do not show 
TABLE 3: NEUROPATHIC PAIN SYMPTOM INVENTORY

\begin{tabular}{|c|c|c|c|c|}
\hline Total intensity score & Sub score & Amitriptyilline & Pregabalin & $\mathrm{P}$ value \\
\hline Q1 & $\begin{array}{l}\text { Burning (superficial) spontaneous pain: } \\
\qquad 1=/ 10\end{array}$ & 3.76 & 3.80 & .002 \\
\hline (Q2+Q3) & $\begin{array}{l}\text { Pressing (deep) spontaneous pain: } \\
\qquad(\mathrm{Q} 2+\mathrm{Q} 3) / 2=/ 10\end{array}$ & 1.11 & 1.40 & 0.070 \\
\hline (Q5+Q6) & $\begin{array}{l}\text { Paroxysmal pain: } \\
(\mathrm{Q} 5+\mathrm{Q} 6) / 2=/ 10\end{array}$ & 3.44 & 2.60 & 0.088 \\
\hline (Q8+Q9+Q10) & $\begin{array}{c}\text { Evoked pain: } \\
(\mathrm{Q} 8+\mathrm{Q} 9+\mathrm{Q} 10) / 2=/ 10\end{array}$ & 1.44 & 1.19 & 0.059 \\
\hline (Q11+Q12) & $\begin{array}{c}\text { Paraesthesia/dysaesthesia: } \\
(\mathrm{Q} 11+\mathrm{Q} 12) / 2=/ 10\end{array}$ & 2.44 & 4.00 & 0.499 \\
\hline \multicolumn{2}{|c|}{ NPSI mean difference pain score } & 12.22 & 13.01 & 0.749 \\
\hline \multicolumn{2}{|c|}{ VAS mean difference pain score } & 6.34 & 5.7 & 0.043 \\
\hline
\end{tabular}

For $n=35$ for each treatment group, 0.043 is the P-value that shows significant difference between the visual analogue scale (VAS) mean difference pain scores. NPSI: neuropathic pain symptom inventory

TABLE 4: ADVERSE EVENTS OBSERVED IN THE STUDY

\begin{tabular}{ccccccc}
\hline Drug & Gender & Dizziness & Somnolence & Daytime sleepiness & Dry mouth & Peripheral oedema \\
\hline \multirow{2}{*}{ Amitriptyline* $^{*}$} & Male $(\mathrm{n}=5)$ & $4(80 \%)$ & $1(20 \%)$ & $5(100 \%)$ & $1(20 \%)$ & - \\
& Female $(\mathrm{n}=6)$ & $3(50 \%)$ & $1(16 \%)$ & $4(66 \%)$ & $2(33 \%)$ & - \\
\multirow{2}{*}{ Pregabalin* $^{*}$} & Male $(\mathrm{n}=6)$ & $3(50 \%)$ & & $4(66 \%)$ & - & $1(16 \%)$ \\
& Female $(\mathrm{n}=6)$ & $2(33 \%)$ & & $5(83 \%)$ & - & $1(16 \%)$ \\
\hline
\end{tabular}

*Denotes the same patient may report for more than one adverse event of the drug

an extensive difference. The greater incidence of ADRs in our study compared to Bhansal et al. was due to the more number of patients recruited.

Cost effectiveness of amitriptyline and pregabalin was measured using the average costs of various brands of amitriptyline (₹ 1.67) and pregabalin (₹ 9.01) and the VAS mean difference scores. VAS scores were chosen over NPSI scores to calculate ICER because there was a statistical difference in efficacy between amitriptyline and pregabalin. The ICER of amitriptyline to pregabalin is -11.46 .

ICER suggests that amitriptyline dominates pregabalin by what is referred to as "extended dominance" because it is both more effective than pregabalin and has a lower cost for the additional effectiveness achieved. There were limitations for this study; first, the subjective difference of pain in patients. Second, the difficulty in obtaining pain scores in elderly and illiterate patients. Other limitations are short duration of study and difficulty in tracking down the patients during follow up.

Based on the results of the study we can conclude that amitriptyline and pregabalin showed statistically significant decrease in VAS and NPSI after therapy. But the mean difference VAS score of amitriptyline has shown significantly better efficacy than pregabalin. Further long duration studies covering large population are needed to validate the data.

\section{Acknowledgements:}

The authors sincerely thank Dr. M. Ramanathan, Principal, PSG College of Pharmacy for support. We also extend our thanks to Dr. R. Senthil Kumar and Dr. R. Suresh Prabhu of Endocrinology Department, PSG Hospitals for their kind help during the study.

\section{Financial assistance:}

None.

\section{Conflict of interests:}

None declared.

\section{REFERENCES}

1. Lisa AK, Craig W. Endocrine Disorders: Diabetes Mellitus. In: Brian KA, Robin LC, Michael EE, Joseph BG, Pamal AJ, Wayne AK, Bradely RW, editors. Applied Therapeutics: The Clinical Use of Drugs. $10^{\text {th }}$ ed. Philadelphia: Wolters Kluwer Press; 2013. p. 1223-25.

2. Jennifer DS. Diabetes Mellitus. In: Leon S, Alan HM, Paul FS, Larry NS, editors. Comprehensive Pharmacy Review. $7^{\text {th }}$ ed. Philadelphia: Wolters Kluwer Press; 2013. p. 930-53.

3. Umesh M, Michael SG. Pancreatic Hormones and Diabetes Mellitus: Specific Chronic Complications of Diabetes Mellitus. In: David GG, Dolores S, editors. Greenspan's Basic and Clinical Endocrinology. 9th ed. San Francisco: McGrawHill Companies; 2007. p. 730-32.

4. Bhansal D, Bhansali A, Hota D, Chakrabarti A, Dutta P. Amitriptyline vs. pregabalin in painful diabetic neuropathy: randomized double blind clinical trial. Diabet Med 2009;26:1019-26. 
5. Neelima R, Dipika B, Shiva TT, Kapil G, Debasish H, Anil B, et al. Comparative efficacy and safety of six antidepressants and anticonvulsants in painful diabetic neuropathy: a network meta-analysis. Pain Physician 2013;16;E705-14.

6. Bouhassira D, Attal N, Fermanian J, Alchaar H, Gautron $\mathrm{M}$, Masquelier $\mathrm{E}$, et al. Development and validation of the Neuropathic Pain Symptom Inventory. Pain 2004;108(3):248-57.

7. Antonieta ML, Ruben MM, Dyfrig H. Approaches to pharmacoeconomic analysis, In: Tom W, Alan H, Angela B, editors. Pharmacoeconomics.1st ed. London: Churchill Livingstone; 2004. p. 110-13. 